\title{
The Compressible Navier-Stokes Equations with Weak Viscosity and Heat Conductivity
}

\author{
Wan Zhang*, Hang Yang, Liping Liu \\ Department of Mathematics, Jinan University, Guangzhou, China \\ Email: *endwan@stu2017.jnu.edu.cn
}

How to cite this paper: Zhang, W., Yang, H. and Liu, L.P. (2019) The Compressible Navier-Stokes Equations with Weak Viscosity and Heat Conductivity. American Journal of Computational Mathematics, 9, 32-47.

https://doi.org/10.4236/ajcm.2019.92003

Received: April 13, 2019

Accepted: May 24, 2019

Published: May 27, 2019

Copyright $\odot 2019$ by author(s) and Scientific Research Publishing Inc. This work is licensed under the Creative Commons Attribution International License (CC BY 4.0).

http://creativecommons.org/licenses/by/4.0/

\begin{abstract}
It is well known that the full compressible Navier-Stokes equations with viscosity and heat conductivity coefficients of order of the Knudsen number $\epsilon>0$ can be deduced from the Boltzmann equation via the Chapman-Enskog expansion. In this paper, we carry out the rigorous mathematical study of the compressible Navier-Stokes equation with the initial-boundary value problems. We construct the existence and most importantly obtain the higher regularities of the solutions of the full compressible Navier-Stokes system with weak viscosity and heat conductivity in a general bounded domain.
\end{abstract}

\section{Keywords}

Compressible Navier-Stokes System, Energy Estimate, the Helmholtz

Decomposition, Elliptic Estimates, the Galerkin Method

\section{Introduction}

This paper is concerned with the following initial boundary value problem of the full compressible Navier-Stokes equations [1] in a smooth bounded domain $\Omega \in \mathbb{R}^{3}$

$$
\left\{\begin{array}{l}
\partial_{t} \rho+\nabla_{x} \cdot(\rho u)=0 \\
\rho\left(\partial_{t} u+u \cdot \nabla_{x} u\right)+\nabla_{x} P=\epsilon\{\mu \Delta u+(\lambda+\mu) \nabla \nabla \cdot u\}, P=\rho \theta, \\
\frac{3}{2} \rho\left(\partial_{t} \theta+u \cdot \nabla_{x} \theta\right)+P \nabla_{x} \cdot u=\epsilon\left\{\kappa \Delta \theta+\frac{\mu}{2}\left((\nabla u)+(\nabla u)^{\mathrm{T}}\right)^{2}+\lambda(\nabla \cdot u)^{2}\right\},
\end{array}\right.
$$

with the initial data

$$
[\rho, u, \theta](0, x)=\left[\rho_{0}, u_{0}, \theta_{0}\right](x),
$$

and the Dirichlet boundary condition

$$
\left.[u, \theta]\right|_{\partial \Omega}=[0,1],
$$


where $\rho(t, x)>0, u(t, x)=\left(u_{1}, u_{2}, u_{3}\right)$ and $\theta(t, x)>0$ denote the density, velocity and temperature of the fluid, respectively. $\epsilon>0$ is the Knudsen number, and the constants $\mu, \lambda>0$ and $\kappa>0$ stand for the viscosity and heat conductivity coefficients, respectively, moreover $\mu$ and $\lambda$ satisfy the physical restrictions $\mu+\frac{2}{3} \lambda>0$. Our goal in this paper is to study the global existence of (1) with $\epsilon>0$ and small enough.

The Navier-Stokes equations are a fundamental model aimed at describing the motion of an incompressible viscous fluid. There are huge number of literatures on the mathematical studies for the compressible Navier-Stokes equations. Here, we only mention the works related to our current study. The full compressible Navier-Stokes equations with viscosity and heat conductivity coefficients of order of the Knudsen number $\epsilon>0$ were formally derived by Bardos-Golse-Levermore [2]. Recently, Liuyang Zhao [3] and Duan Liu [4] justified the compressible Navier-Stokes equations as the second order approximation to the Boltzmann equation in the whole space and in general bounded domain, respectively. Wang [5] and Wang Xinyong [6] studied the vanishing viscosity limits of the compressible Navier-Stokes system, while the same issue for the incompressible one was also investigated by Masmoudi-Rousset [7]. When the viscosity and heat conductivity are independent of the Kundsen number, Matsumura-Nishida constructed the global existence in the whole space [8] and in bounded domain [9] by an elementary energy method, respectively. Recently, Huang Lixin [10] proved the global well-posedness of classical solutions with large oscillations and vacuum for the isentropic compressible Navier-Stokes equations. The large time behaviors of the classical solutions are studied by Ukai-Yang-Zhao [11] and Duan-Liu-Ukai-Yang [12] and reference therein. For the mathematical study of the weak solutions of the compressible Navier-Stokes equations we refer to the survey book by Lions [13].

Compared with the previous works such as [8] and [9], the main difficulty in the current paper is the weak dissipation structure of the system (1), say, the coefficients of the viscosity and heat conductivity involve the Knudsen number, it is quite hard to establish the higher regularities of the solutions. More precisely, the standard elliptic estimates cannot be directly applied here due to the singularity perturbation and the usual regularity estimates to deal with the boundary value problem of elliptic partial differential equations which cannot be available either. To overcome those difficulties, we introduce the Helmholtz decomposition, the Galerkin method and conormal derivatives to handle the boundary terms and obtain the higher order energy estimates. The overall structure of this paper is to make zero-order, first-order, second-order energy estimates and conormal energy estimates for Navier-Stokes, we construct the existence and most importantly obtain the higher regularities of the solutions of the full compressible Navier-Stokes system with weak viscosity and heat conductivity in a general bounded domain.

Notations. Throughout this paper, $C$ denotes some generic positive (generally 
large) constant and $\lambda$ denotes some generic positive (generally small) constants, where $C$ and $\lambda$ may take different values in different places. $D \lesssim E$ means that there is a generic constant $C>0$ such that $D \leq C E$.

\section{Solutions of the Compressible Navier-Stokes Equations}

This section is devoted to proving the global classical solution to the initial boundary value problem of (1), (2) and (3), the main result of this paper. We emphasize that it is extremely difficult to obtain the uniform higher regularity of the solutions of the system (1), (2) and (3) due to the weak dissipation on the right hand side and the non-slip boundary condition, which is quite different from the incompressible case, where the standard elliptic estimates can be directly adopted to gain the regularity of the solutions, cf. [7]. To settle this problem, it is convenient to introduce the so-called conormal derivatives. Since $\partial \Omega$ is compact, one can find finitely many points $x_{i}^{0} \in \partial \Omega$, radii $r_{i}>0$, corresponding sets $\Omega_{i}=\Omega \cap B^{0}\left(x_{i}^{0}, r_{i}\right)$ and smooth functions $\phi_{i} \in C^{k}\left(\bar{\Omega}_{i}\right)$ $(i=1,2, \cdots, m, k \geq 6)$ such that $\partial \Omega \subset \bigcup_{i}^{m} B^{0}\left(x_{i}^{0}, r_{i}\right)$ and

$$
\Omega_{i}=\left\{x \in B^{0}\left(x_{i}^{0}, r_{i}\right) \mid x_{3}>\phi_{i}\left(x_{1}, x_{2}\right)\right\}, m \geq i \geq 1 .
$$

In what follows, we omit the subscript $i$ of $\phi_{i}$ for notational simplicity. Using this, we now change coordinates so as to flatten out the boundary. To be more specific, we define

$$
\Phi:(y, z) \mapsto(y, \phi(y)+z)=x .
$$

Denoting $e_{y^{1}}=\left(1,0, \partial_{1} \phi\right)^{\mathrm{T}}, e_{y^{2}}=\left(0,1, \partial_{2} \phi\right)^{\mathrm{T}}$ and $e_{z}=(0,0,-1)^{\mathrm{T}}$, one sees that $\left(e_{y^{1}}, e_{y^{2}}, e_{z}\right)$ is a local basis around the boundary. We emphasize that $e_{y^{1}}$ and $e_{y^{2}}$ on the boundary are tangent to $\partial \Omega$, and in general, $e_{z}$ is not a normal vector field.

The following lemma is concerned with the basic properties of the conormal derivatives.

Lemma 1. Let

$$
Z_{i}=\partial_{y^{i}}=\partial_{i}-\partial_{i} \phi \partial_{z}, i=1,2,
$$

where $\varphi(z)=\frac{z}{1+z}$ is smooth, supported in $\mathbb{R}_{+}$with the property $\varphi(0)=0$, $\varphi^{\prime}(0)>0, \varphi(z)>0$ for $z>0$. It is easy to check that

$$
Z_{1} Z_{2}=Z_{2} Z_{1} \text {, }
$$

and

$$
\partial_{z} Z_{i}=Z_{i} \partial_{z}, i=1,2 \text {. }
$$

We now define the following Sobolev conormal derivatives

$$
Z^{\alpha}=\partial_{t}^{\alpha_{0}} \mathcal{Z}^{\alpha_{1}}=\partial_{t}^{\alpha_{0}} Z_{1}^{\alpha_{11}} Z_{2}^{\alpha_{12}},
$$

where $\alpha, \alpha_{0}, \alpha_{1}$ are the differential multi-indices with $\alpha \stackrel{\text { def }}{=}\left(\alpha_{0}, \alpha_{1}\right), \alpha_{1}=\left(a_{11}, \alpha_{12}\right)$, and the corresponding Sobolev conormal norm: 


$$
\|f(t)\|_{H_{c o}^{m}}^{2}=\sum_{|\alpha| \leq m}\left\|Z^{\alpha} f(t)\right\|_{L_{x}^{2}}^{2},\|f(t)\|_{H_{c o}^{k, \infty}}=\sum_{|\alpha| \leq k}\left\|Z^{\alpha} f(t)\right\|_{L_{x}^{\infty}}
$$

for smooth function $f(t, x)$. Note that we also use $H^{k}$ to denote the usual Sobolev space $W^{k, 2}(\Omega)$.

The following anisotropic Sobolev embedding and trace estimates which are given in [[7], Proposition 2.2, pp. 316] will be frequently used in the later proof.

Lemma 2. Let $m_{1} \geq 0, m_{2} \geq 0$ be integers, $f \in H_{c o}^{m_{1}}(\Omega) \cap H_{c o}^{m_{2}}(\Omega)$ and $\nabla f \in H_{c o}^{m_{2}}(\Omega)$.

Then

$$
\|f\|_{L^{\infty}}^{2} \leq C\|\nabla f\|_{H_{c o}^{m_{2}}}\|f\|_{H_{c o}^{m_{1}}}
$$

provided $m_{1}+m_{2} \geq 3$, and

$$
|f|_{H^{s}(\partial \Omega)}^{2} \leq C\left(\|\nabla f\|_{H_{c o}^{m_{2}}}+\|f\|_{H_{c o}^{m_{2}}}\right)\|f\|_{H_{c o}^{m_{1}}}
$$

for $m_{1}+m_{2} \geq 2 s \geq 0$.

The solution of (1), (2) and (3) is sought in the set of the functions space

$$
\mathbf{X}_{\epsilon}(t)=\left\{[\rho, u, \theta]\left|\|[\rho-1, u, \theta-1]\|_{\mathbf{X}_{\epsilon}}^{2} \leq c_{0} \epsilon^{2}, c_{0}>0,2 \alpha_{0}+\right| \alpha_{1} \mid \leq m_{0}, m_{0} \geq 6\right\},
$$

where

$$
\begin{aligned}
\|[\rho-1, u, \theta-1]] \|_{\mathbf{X}_{\epsilon}}^{2}= & \sup _{0 \leq s \leq t}\left\{\|[\rho-1, u, \theta-1](s)\|_{H_{c o}^{m_{0}}}^{2}+\|\nabla[\rho, u, \theta](s)\|_{H_{c o}^{m_{0}-1}}^{2}\right\} \\
& +\sup _{0 \leq s \leq t}\left\{\epsilon^{2}\left\|\nabla^{2}[\rho, u, \theta]\right\|_{H_{c o}^{m_{0}-2}}^{2}+\epsilon^{4}\left\|\nabla^{3}[u, \theta]\right\|_{H_{c o}^{m_{0}-3}}^{2}\right\} .
\end{aligned}
$$

We now state the main result of this paper.

Theorem 1. Let $\kappa_{0}>0$ and $m_{0} \geq 6$. If

$$
\left\|\left[\rho_{0}-1, u_{0}, \theta_{0}-1\right]\right\|_{\mathbf{X}_{\epsilon}} \leq \kappa_{0} \epsilon
$$

then there exists a unique global smooth solution $[\rho, u, \theta](t, x)$ to (1), (2) and (3) satisfying

$$
\begin{aligned}
& \|[\rho-1, u, \theta-1]\|_{H_{c o}^{m_{0}}}^{2}+\left\|\nabla_{x}[\rho, u, \theta]\right\|_{H_{c o}^{m_{0}-1}}^{2}+\epsilon^{2}\left\|\nabla_{x}^{2}[\rho, u, \theta]\right\|_{H_{c o}^{m_{0}-2}}^{2} \\
& +\epsilon^{4}\left\|\nabla_{x}^{3}[u, \theta]\right\|_{H_{c o}^{m_{0}-3}}^{2}+\epsilon \int_{0}^{t}\left\|\nabla_{x} \rho\right\|_{H_{c o}^{m_{0}-1}}^{2} \mathrm{~d} s+\epsilon \int_{0}^{t}\left\|\nabla_{x}[u, \theta]\right\|_{H_{c o}^{m_{0}}}^{2} \mathrm{~d} s \\
& +\epsilon \int_{0}^{t}\left\|\nabla_{x}^{2} \rho\right\|_{H_{c o}^{m_{0}-2}}^{2} \mathrm{~d} s+\epsilon \int_{0}^{t}\left\|\nabla_{x}^{2}[u, \theta]\right\|_{H_{c o}^{m_{0}-1}}^{2} \mathrm{~d} s \\
& \leq C_{0}\left\|\left[\rho_{0}-1, u_{0}, \theta_{0}-1\right]\right\|_{\mathbf{X}_{\epsilon}}^{2}
\end{aligned}
$$

for $C_{0}>0$.

Proof. The local existence of (1), (2) and (3) follows from a standard iteration method, we only prove the a priori estimate (4) under the a priori assumption

$$
N(t) \leq \kappa_{0}^{2} \epsilon^{2}
$$

where $N(t)$ is given by 


$$
\begin{aligned}
N(t)= & N(\rho, u, \theta)(t) \\
= & \|[\rho-1, u, \theta-1]\|_{H_{c o}^{m_{0}}}^{2}+\left\|\nabla_{x}[\rho, u, \theta]\right\|_{H_{c o}^{m_{0}-1}}^{2}+\epsilon^{2}\left\|\nabla_{x}^{2}[\rho, u, \theta]\right\|_{H_{c o}^{m_{0}-2}}^{2} \\
& +\epsilon^{4}\left\|\nabla_{x}^{3}[u, \theta]\right\|_{H_{c o}^{m_{0}-3}}^{2}+\epsilon \int_{0}^{t}\left\|\nabla_{x} \rho\right\|_{H_{c o}^{m_{0}-1}}^{2} \mathrm{~d} s+\epsilon \int_{0}^{t}\left\|\nabla_{x}[u, \theta]\right\|_{H_{c o}^{m_{0}}}^{2} \mathrm{~d} s \\
& +\epsilon \int_{0}^{t}\left\|\nabla_{x}^{2} \rho\right\|_{H_{c o}^{m_{0}-2}} \mathrm{~d} s+\epsilon \int_{0}^{t}\left\|\nabla_{x}^{2}[u, \theta]\right\|_{H_{c o}^{m_{0}-1}}^{2} \mathrm{~d} s .
\end{aligned}
$$

The proof is then divided into the following four steps.

Step 1. The zeroth order energy estimate. Denote $[\tilde{\rho}, \tilde{\theta}]=[\rho-1, \theta-1]$, take the inner product of $(1)_{1},(1)_{2}$ and $(1)_{3}$ with $\tilde{\rho}, u$ and $\frac{\tilde{\theta}}{\theta}$, respectively, to obtain

$$
\begin{aligned}
& \frac{1}{2} \frac{\mathrm{d}}{\mathrm{d} t}\|\tilde{\rho}\|_{2}^{2}+\left(\nabla_{x} \tilde{\rho} u, \tilde{\rho}\right)+\left(\tilde{\rho} \nabla_{x} \cdot u, \tilde{\rho}\right)+\left(\nabla_{x} \cdot u, \tilde{\rho}\right)=0, \\
& \frac{1}{2} \frac{\mathrm{d}}{\mathrm{d} t} \int_{\Omega} \rho u^{2} \mathrm{~d} x+\left(\nabla_{x} \tilde{\rho}, u\right)+\left(\nabla_{x} \tilde{\theta}, u\right)+\left(\tilde{\theta} \nabla_{x} \tilde{\rho}, u\right)+\left(\tilde{\rho} \nabla_{x} \tilde{\theta}, u\right) \\
&=-\mu \epsilon\left\|\nabla_{x} u\right\|_{2}^{2}-(\lambda+\mu) \epsilon\left\|\nabla_{x} \cdot u\right\|_{2}^{2},
\end{aligned}
$$

$\frac{3}{4} \frac{\mathrm{d}}{\mathrm{d} t} \int_{\Omega} \frac{\rho}{\theta} \tilde{\theta}^{2} \mathrm{~d} x+\frac{3}{4} \int_{\Omega} \frac{\rho}{\theta^{2}} \tilde{\theta}^{2} \partial_{t} \theta \mathrm{d} x+\frac{3}{4} \int_{\Omega} \frac{\rho}{\theta^{2}} \tilde{\theta}^{2} u \cdot \nabla_{x} \theta \mathrm{d} x+\left(\nabla_{x} \cdot u, \tilde{\theta}\right)+\left(\tilde{\rho} \nabla_{x} \cdot u, \tilde{\theta}\right)$

$=-\kappa \epsilon\left\|\frac{\nabla_{x} \tilde{\theta}}{\sqrt{\theta}}\right\|_{2}^{2}+\kappa \epsilon\left\|\frac{\sqrt{\tilde{\theta}}}{\theta} \nabla_{x} \theta\right\|_{2}^{2}+\frac{1}{2} \mu \epsilon\left((\nabla u)+(\nabla u)^{\mathrm{T}}\right)^{2}+\lambda \epsilon\left((\nabla \cdot u)^{2}, \frac{\tilde{\theta}}{\theta}\right)$.

Taking the summation of (6), (7) and (8), applying Lemma 2 and the a priori assumption (5), we then have for some $\lambda>0$

$$
\begin{aligned}
& \|[\tilde{\rho}, u, \tilde{\theta}](t)\|_{2}^{2}+\lambda \epsilon \int_{0}^{t}\left\|\nabla_{x}[u, \tilde{\theta}](s)\right\|_{2}^{2} \mathrm{~d} s \\
& \leq C\|[\tilde{\rho}, u, \tilde{\theta}](0, x)\|_{2}^{2}+\kappa_{0} \epsilon \int_{0}^{t}\left\|\nabla_{x}[\tilde{\rho}, u, \tilde{\theta}](s)\right\|_{2}^{2} \mathrm{~d} s .
\end{aligned}
$$

To obtain the dissipation of $\nabla_{x} \tilde{\rho}$, we next get from the inner product of $\epsilon\left((1)_{1}, \nabla_{x} \cdot u\right)$ and $\epsilon\left((2)_{2}, \nabla_{x} \tilde{\rho} / \rho\right)$ that

$$
\begin{aligned}
& \int_{0}^{t} \epsilon\left(\partial_{t} \tilde{\rho}, \nabla_{x} \cdot u\right) \mathrm{d} s+\int_{0}^{t} \epsilon\left(\nabla_{x} \cdot(\rho u), \nabla_{x} \cdot u\right) \mathrm{d} s-\int_{0}^{t} \epsilon \frac{\mathrm{d}}{\mathrm{d} s}\left(u, \nabla_{x} \tilde{\rho}\right) \mathrm{d} s \\
& +\int_{0}^{t} \epsilon\left(u, \nabla_{x} \partial_{t} \rho\right) \mathrm{d} s-\int_{0}^{t} \epsilon\left(u \cdot \nabla_{x} u, \nabla_{x} \tilde{\rho}\right) \mathrm{d} s-\int_{0}^{t} \epsilon\left(\nabla_{x} P, \nabla_{x} \tilde{\rho} / \rho\right) \mathrm{d} s \\
& =-\int_{0}^{t} \epsilon^{2}\left(\mu \Delta u+(\lambda+\mu) \nabla \nabla \cdot u, \nabla_{x} \tilde{\rho} / \rho\right) \mathrm{d} s \\
& \Leftrightarrow \int_{0}^{t} \epsilon\left(\nabla_{x} \cdot(\rho u), \nabla_{x} \cdot u\right) \mathrm{d} s-\int_{0}^{t} \epsilon \frac{\mathrm{d}}{\mathrm{d} s}\left(u, \nabla_{x} \tilde{\rho}\right) \mathrm{d} s \\
& \quad-\int_{0}^{t} \epsilon\left(u \cdot \nabla_{x} u, \nabla_{x} \tilde{\rho}\right) \mathrm{d} s-\int_{0}^{t} \epsilon\left(\nabla_{x} P, \nabla_{x} \tilde{\rho} / \rho\right) \mathrm{d} s \\
& =-\int_{0}^{t} \epsilon^{2}\left(\mu \Delta u+(\lambda+\mu) \nabla \nabla \cdot u, \nabla_{x} \tilde{\rho} / \rho\right) \mathrm{d} s,
\end{aligned}
$$

where we used the fact $\left(u, \nabla_{x} \partial_{t} \rho\right)+\left(\nabla_{x} u, \partial_{t} \tilde{\rho}\right)=0$. The above identity then implies

$$
\begin{aligned}
& -\epsilon\left(u, \nabla_{x} \tilde{\rho}\right)(t)+\lambda \epsilon \int_{0}^{t}\left\|\nabla_{x} \tilde{\rho}\right\|_{2}^{2} \mathrm{~d} s \\
& \leq C \epsilon\left|\left(u, \nabla_{x} \tilde{\rho}\right)(0)\right|+C_{\eta} \epsilon^{3} \int_{0}^{t}\left\|\nabla_{x}^{2} u\right\|_{2}^{2} \mathrm{~d} s+C\left(\epsilon+\epsilon^{2}\right) \int_{0}^{t}\left\|\nabla_{x}[u, \tilde{\theta}](s)\right\|_{2}^{2} \mathrm{~d} s \\
& +C\left(\kappa_{0}+\eta\right) \epsilon \int_{0}^{t}\left\|\nabla_{x} \tilde{\rho}\right\|_{2}^{2} \mathrm{~d} s,
\end{aligned}
$$


Let $\kappa_{0}$ and $\epsilon$ be suitably small, then (9) and (10) give rise to

$$
\begin{aligned}
& \|[\tilde{\rho}, u, \tilde{\theta}](t)\|_{2}^{2}-\epsilon\left|\left(u, \nabla_{x} \tilde{\rho}\right)\right|+\lambda \epsilon \int_{0}^{t}\left\|\nabla_{x}[\tilde{\rho}, u, \tilde{\theta}](s)\right\|_{2}^{2} \mathrm{~d} s \\
& \leq C\left\|\left[\tilde{\rho}_{0}, u_{0}, \tilde{\theta}_{0}\right]\right\|_{2}^{2}+C \epsilon\left|\left(u_{0}, \nabla_{x} \tilde{\rho}_{0}\right)\right|+C \tilde{\theta}^{3} \int_{0}^{t}\left\|\nabla_{x}^{2} u\right\|_{2}^{2} \mathrm{~d} s \\
& \leq C N(0)+C \epsilon^{2} N(t) .
\end{aligned}
$$

Similarly, by acting $\partial_{t}^{\alpha_{0}} \quad\left(\alpha_{0} \leq\left[m_{0} / 2\right]\right)$ to (1), one also has

$$
\begin{aligned}
& \left\|\partial_{t}^{\alpha_{0}}[\tilde{\rho}, u, \tilde{\theta}](t)\right\|_{2}^{2}-\epsilon\left|\left(\partial_{t}^{\alpha_{0}} u, \partial_{t}^{\alpha_{0}} \nabla_{x} \tilde{\rho}\right)\right|+\lambda \epsilon \int_{0}^{t}\left\|\nabla_{x} \partial_{t}^{\alpha_{0}}[\tilde{\rho}, u, \tilde{\theta}](s)\right\|_{2}^{2} \mathrm{~d} s \\
& \leq C\left\|\partial_{t}^{\alpha_{0}}\left[\tilde{\rho}_{0}, u_{0}, \tilde{\theta}_{0}\right]\right\|_{2}^{2}+C \epsilon\left|\left(\partial_{t}^{\alpha_{0}} u_{0}, \nabla_{x} \partial_{t}^{\alpha_{0}} \tilde{\rho}_{0}\right)\right|+C \epsilon^{3} \int_{0}^{t}\left\|\nabla_{x}^{2} \partial_{t}^{\alpha_{0}} u\right\|_{2}^{2} \mathrm{~d} s \\
& \leq C N(0)+C \epsilon^{2} N(t) .
\end{aligned}
$$

Step 2. The first order energy estimate. The energy estimates for $\nabla_{x}[\tilde{\rho}, u, \tilde{\theta}]$ are subtle since we know nothing about the derivatives of these quantities on the boundary and the dissipation of (1) is very weak. Our strategy to take care of these difficulties is the Helmholtz decomposition, elliptic estimates and the Galerkin method. To see this, we first decompose $u$ as $u=u^{1}+u^{2}$ with $u^{1}=\nabla_{x} \mathbf{u}, u^{2}=\nabla_{x} \times \mathbb{V}$ and $\left.u^{2}\right|_{\partial \Omega}=0$.

Moreover, we set $\theta_{m}(t, x)-1=\sum_{k=1}^{m} d_{k}(t) \mathbb{W}_{k}(x)$ with $\mathbb{W}_{k}(x) \in H_{0}^{1}(\Omega)(k=1,2, \cdots)$ being the eigenvalues of the operator $-\Delta_{x}$, i.e.

$$
\left\{\begin{array}{l}
-\Delta_{x} \mathbb{W}_{k}=\lambda_{k} \mathbb{W}_{k}, x \in \Omega, \\
\mathbb{W}_{k}=0, x \in \partial \Omega
\end{array}\right.
$$

where $0<\lambda_{1} \leq \lambda_{2} \leq \cdots$. The key point here is that we get an approximation sequence $\theta_{m}$ such that $\left.\Delta \theta_{m}\right|_{\partial \Omega}=0$.

We now approximate (1) as

$$
\left\{\begin{array}{l}
\partial_{t} \rho+\nabla_{x} \cdot(\rho u)=0, \\
\rho\left(\partial_{t} u+u \cdot \nabla_{x} u\right)+\nabla_{x}\left(\rho \theta_{m}\right)=\epsilon\{\mu \Delta u+(\lambda+\mu) \nabla \nabla \cdot u\}, \\
\frac{3}{2} \rho\left(\partial_{t} \theta_{m}+u \cdot \nabla_{x} \theta_{m}\right)+\rho \theta_{m} \nabla_{x} \cdot u=\epsilon\left\{\kappa \Delta \theta+\frac{\mu}{2}\left((\nabla u)+(\nabla u)^{\mathrm{T}}\right)^{2}+\lambda(\nabla \cdot u)^{2}\right\}, \\
{\left[\rho, u, \theta_{m}\right](0, x)=\left[\rho_{0}, u_{0}, \theta_{0}\right](x),} \\
{\left.\left[u, \theta_{m}\right]\right|_{\partial \Omega}=[0,1] .}
\end{array}\right.
$$

Note that here $[\rho, u] \stackrel{\text { def }}{=}\left[\rho_{m}, u_{m}\right]$ also depend on $m$, we drop the subscript $m$ for brevity.

Noticing $\nabla_{x} \cdot u^{2}=0, \nabla_{x} \times u^{1}=0$ and $\left(u^{1}, u^{2}\right)=0$, taking the inner product of $(12)_{2}$ with $\partial_{t} u^{2}$ and integrating the resulting equation with respect to $t$, one has

$$
\begin{aligned}
& \epsilon\left(\nabla_{x} \times u^{2}, \nabla_{x} \times u^{2}\right)+\lambda \int_{0}^{t}\left\|\partial_{t} u^{2}\right\|_{2}^{2} \mathrm{~d} s \\
& \leq C \epsilon\left|\left(\nabla_{x} \times u_{0}^{2}, \nabla_{x} \times u_{0}^{2}\right)\right|+C \int_{0}^{t}\left\|\tilde{\rho} \partial_{t} u\right\|_{2}^{2} \mathrm{~d} s+C \int_{0}^{t}\left\|\rho u \cdot \nabla_{x} u\right\|_{2}^{2} \mathrm{~d} s \\
& \leq C \epsilon\left|\left(\nabla_{x} \times u_{0}^{2}, \nabla_{x} \times u_{0}^{2}\right)\right|+C \kappa_{0}^{2} \epsilon^{2} \int_{0}^{t}\left\|\nabla_{x}[\tilde{\rho}, u](s)\right\|_{2}^{2} \mathrm{~d} s \\
& \leq C \epsilon N(0)+C \kappa_{0}^{2} \epsilon N(t),
\end{aligned}
$$


here the fact that $\Delta u=\nabla_{x} \nabla_{x} \cdot u-\nabla_{x} \times \nabla_{x} \times u$ and $\left(\nabla_{x} \times \nabla_{x} \times u_{2}, \partial_{t} u_{2}\right)=\left(\nabla_{x} \times u_{2}, \nabla_{x} \times \partial_{t} u_{2}\right)$ was used.

Likewise, it follows that for $\alpha_{0} \leq\left[\frac{m_{0}-1}{2}\right]$

$$
\begin{aligned}
& \epsilon\left(\nabla_{x} \times \partial_{t}^{\alpha_{0}} u^{2}, \nabla_{x} \times \partial_{t}^{\alpha_{0}} u^{2}\right)+\lambda \int_{0}^{t}\left\|\partial_{t}^{\alpha_{0}+1} u^{2}\right\|_{2}^{2} \mathrm{~d} s \\
& \leq C \epsilon\left|\left(\nabla_{x} \times \partial_{t}^{\alpha_{0}} u_{0}^{2}, \nabla_{x} \times \partial_{t}^{\alpha_{0}} u_{0}^{2}\right)\right|+C \kappa_{0}^{2} \epsilon^{2} \int_{0}^{t} \mid \nabla_{x} \partial_{t}^{\alpha_{0}}[\tilde{\rho}, u](s) \|_{2}^{2} \mathrm{~d} s \\
& \leq C \epsilon N(0)+C \kappa_{0}^{2} \epsilon N(t) .
\end{aligned}
$$

Next, we consider the following elliptic problems

$$
\left\{\begin{array}{l}
\epsilon \nabla_{x} \cdot u=-\epsilon \partial_{t} \rho-\epsilon \tilde{\rho} \nabla_{x} \cdot u-\epsilon \nabla_{x} \rho \cdot u \stackrel{\text { def }}{=} h_{1}, \\
-\mu \epsilon \Delta u+\nabla_{x} \bar{P}=-\partial_{t} u^{2}-\tilde{\rho} \partial_{t} u-\rho u \cdot \nabla_{x} u \stackrel{\text { def }}{=} h_{2}, \\
\left.u\right|_{\partial \Omega}=0,
\end{array}\right.
$$

where

$$
\bar{P}=\partial_{t} \mathbf{u}+\rho \theta_{m}-\epsilon(\lambda+\mu) \nabla_{x} \cdot u .
$$

In view of Lemma 4.3 in [[9], pp. 451] and applying Lemma 2, one has for

$$
\begin{aligned}
& \alpha_{0} \leq {\left[\frac{m_{0}-2}{2}\right] } \\
& \epsilon^{2}\left\|\nabla_{x}^{2} \partial_{t}^{\alpha_{0}} u\right\|_{2}^{2} \lesssim\left\|\nabla_{x} \partial_{t}^{\alpha_{0}} h_{1}\right\|_{2}^{2}+\left\|\partial_{t}^{\alpha_{0}} h_{2}\right\|_{2}^{2} \\
&=\left\|\nabla_{x} \partial_{t}^{\alpha_{0}}\left(-\epsilon \partial_{t} \rho-\epsilon \tilde{\rho} \nabla_{x} \cdot u-\epsilon \nabla_{x} \rho \cdot u\right)\right\|_{2}^{2}+\left\|\partial_{t}^{\alpha_{0}}\left(-\partial_{t} u^{2}-\tilde{\rho} \partial_{t} u-\rho u \cdot \nabla_{x} u\right)\right\|_{2}^{2} \\
& \lesssim \epsilon^{2}\left\|\partial_{t}^{\alpha_{0}+1} \nabla_{x} \tilde{\rho}\right\|_{2}^{2}+\epsilon^{2}\left\|\nabla_{x} \partial_{t}^{\alpha_{0}}\left(\tilde{\rho} \nabla_{x} \cdot u\right)\right\|_{2}^{2}+\epsilon^{2}\left\|\nabla_{x} \partial_{t}^{\alpha_{0}}\left(\nabla_{x} \rho \cdot u\right)\right\|_{2}^{2} \\
& \quad+\left\|\partial_{t}^{\alpha_{0}+1} u^{2}\right\|_{2}^{2}+\left\|\partial_{t}^{\alpha_{0}}\left(\tilde{\rho} \partial_{t} u\right)\right\|_{2}^{2}+\left\|\partial_{t}^{\alpha_{0}}\left(\rho u \cdot \nabla_{x} u\right)\right\|_{2}^{2} \\
& \lesssim \epsilon^{2}\left\|\partial_{t}^{\alpha_{0}+1} \nabla_{x} \tilde{\rho}\right\|_{2}^{2}+\kappa_{0}^{2} \epsilon^{3}\left\|\nabla_{x} \partial_{t}^{\alpha_{0}}[\tilde{\rho}, u]\right\|_{2}^{2}+\kappa_{0}^{2} \epsilon^{4}\left\|\nabla_{x}^{2} \partial_{t}^{\alpha_{0}}[\tilde{\rho}, u]\right\|_{2}^{2} \\
&+\left\|\partial_{t} \partial_{t}^{\alpha_{0}} u^{2}\right\|_{2}^{2}+\kappa_{0}^{2} \epsilon^{2}\left\|\partial_{t}^{\alpha_{0}}\left[\nabla_{x} \rho, \nabla_{x} u\right]\right\|_{2}^{2} .
\end{aligned}
$$

From (13), (14) and (11), it follows

$$
\begin{aligned}
& \left(\nabla_{x} \times \partial_{t}^{\alpha_{0}} u^{2}, \nabla_{x} \partial_{t}^{\alpha_{0}} \times u^{2}\right)+\frac{\lambda}{\epsilon} \int_{0}^{t}\left\|\partial_{t} \partial_{t}^{\alpha_{0}} u^{2}\right\|_{2}^{2} \mathrm{~d} s+\lambda \epsilon \int_{0}^{t}\left\|\nabla_{x}^{2} \partial_{t}^{\alpha_{0}} u\right\|_{2}^{2} \mathrm{~d} s \\
& \lesssim C N(0)+C \kappa_{0}^{2} N(t)+\lambda \epsilon \int_{0}^{t}\left\|\partial_{t}^{\alpha_{0}+1} \nabla_{x} \tilde{\rho}\right\| \mathrm{d} s+\lambda \kappa_{0}^{2} \epsilon^{2} \int_{0}^{t}\left\|\nabla_{x} \partial_{t}^{\alpha_{0}}[\tilde{\rho}, u]\right\|_{2}^{2} \mathrm{~d} s \\
& \quad+\lambda \kappa_{0}^{2} \epsilon^{3} \int_{0}^{t}\left\|\nabla_{x}^{2} \partial_{t}^{\alpha_{0}}[\tilde{\rho}, u]\right\|_{2}^{2} \mathrm{~d} s+\lambda \kappa_{0}^{2} \epsilon \lambda \int_{0}^{t}\left\|\partial_{t}^{\alpha_{0}}\left[\nabla_{x} \rho, \nabla_{x} u\right]\right\|_{2}^{2} \mathrm{~d} s \\
& \lesssim C N(0)+C\left(\kappa_{0}^{2}+\epsilon^{2}\right) N(t) .
\end{aligned}
$$

Moreover, by using

$$
\begin{aligned}
-\epsilon \mu \Delta u-\epsilon(\lambda+\mu) \nabla_{x} \nabla_{x} \cdot u=-\rho \partial_{t} u-\rho u \cdot \nabla_{x} u-\nabla_{x} p, \\
-\epsilon \kappa \Delta \theta_{m}=-\frac{3}{2} \rho\left(\partial_{t} \theta_{m}+u \cdot \nabla_{x} \theta_{m}\right)-\rho \theta_{m} \nabla_{x} \cdot u \\
+\epsilon\left\{\frac{\mu}{2}\left(\left(\nabla_{x} u\right)+\left(\nabla_{x} u\right)^{\mathrm{T}}\right)^{2}+\lambda\left(\nabla_{x} \cdot u\right)^{2}\right\},
\end{aligned}
$$


we get from standard elliptic estimates that

$$
\begin{gathered}
\epsilon^{4}\left\|\nabla_{x}^{3} \partial_{t}^{\alpha_{0}} u\right\|_{2}^{2} \lesssim \epsilon^{2}\left\|\partial_{t} \partial_{t}^{\alpha_{0}} u\right\|_{H^{1}}^{2}+\epsilon^{2}\left\|\nabla_{x} \partial_{t}^{\alpha_{0}}\left[\tilde{\rho}, \theta_{m}\right]\right\|_{H^{1}}^{2}+\kappa_{0}^{2} \epsilon^{3}\left\|\nabla_{x} \partial_{t}^{\alpha_{0}} u\right\|_{H^{1}}^{2}, \\
\varepsilon^{2}\left\|\nabla_{x}^{2} \partial_{t}^{\alpha_{0}} \theta_{m}\right\|_{2}^{2} \lesssim\left\|\left[\partial_{t} \partial_{t}^{\alpha_{0}} \theta_{m}, \nabla_{x} \partial_{t}^{\alpha_{0}} u\right]\right\|_{2}^{2}+\kappa_{0}^{2} \epsilon^{2}\left\|\nabla_{x} \partial_{t}^{\alpha_{0}}\left[u, \theta_{m}\right]\right\|_{2}^{2},
\end{gathered}
$$

and

$$
\epsilon^{4}\left\|\nabla_{x}^{3} \partial_{t}^{\alpha_{0}} \theta_{m}\right\|_{2}^{2} \lesssim \epsilon^{2}\left\|\left[\partial_{t} \partial_{t}^{\alpha_{0}} \theta_{m}, \nabla_{x} \partial_{t}^{\alpha_{0}} u\right]\right\|_{H^{1}}^{2}+\kappa_{0}^{2} \epsilon^{4}\left\|\nabla_{x} \partial_{t}^{\alpha_{0}}\left[u, \theta_{m}\right]\right\|_{H^{1}}^{2} .
$$

Next, $\left(\nabla_{x}(12)_{1}, \nabla_{x} \rho\right)-\left((12)_{2}, \nabla_{x} \nabla_{x} \cdot u\right)+\left(\nabla_{x}(12)_{3}, \nabla_{x} \theta_{m}\right)$ yields

$$
\begin{aligned}
& \frac{1}{2} \frac{\mathrm{d}}{\mathrm{d} t}\left\|\nabla_{x} \rho\right\|_{2}^{2}+\frac{1}{2} \frac{\mathrm{d}}{\mathrm{d} t}\left\|\sqrt{\rho} \nabla_{x} \cdot u\right\|_{2}^{2}+\frac{3}{4} \frac{\mathrm{d}}{\mathrm{d} t}\left\|\sqrt{\rho} \nabla_{x} \theta_{m}\right\|_{2}^{2}+\left(\nabla_{x}\left(\nabla_{x} \rho \cdot u\right), \nabla_{x} \rho\right) \\
& +\left(\nabla_{x}\left(\tilde{\rho} \nabla_{x} \cdot u\right), \nabla_{x} \rho\right)-\frac{1}{2}\left(\partial_{t} \rho \nabla_{x} \cdot u, \nabla_{x} \cdot u\right)+\left(\nabla_{x} \rho \cdot \partial_{t} u, \nabla_{x} \cdot u\right) \\
& +\left(\nabla_{x} \cdot\left(\rho u \cdot \nabla_{x} u\right), \nabla_{x} \cdot u\right)-\left(\tilde{\theta}_{m} \nabla_{x} \rho, \nabla_{x} \nabla_{x} \cdot u\right)-\left(\tilde{\rho} \nabla_{x} \theta_{m}, \nabla_{x} \nabla_{x} \cdot u\right) \\
& -\left(\nabla_{x} \theta_{m}, \nabla_{x} \nabla_{x} \cdot u\right)+\epsilon\left(\mu \Delta u+(\lambda+\mu) \nabla_{x} \nabla_{x} \cdot u, \nabla_{x} \nabla_{x} \cdot u\right) \\
& +\frac{3}{2}\left(\nabla_{x} \rho \partial_{t} \theta_{m}, \nabla_{x} \theta_{m}\right)-\frac{3}{4}\left(\partial_{t} \rho \nabla_{x} \theta_{m}, \nabla_{x} \theta_{m}\right)+\frac{3}{2}\left(\nabla_{x}\left(\rho u \cdot \nabla_{x} \theta_{m}\right), \nabla_{x} \theta_{m}\right) \\
& +\left(\nabla_{x}\left(\rho \theta_{m}\right) \nabla_{x} \cdot u, \nabla_{x} \theta_{m}\right)+\left(\tilde{\rho} \nabla_{x} \nabla_{x} \cdot u, \nabla_{x} \theta_{m}\right)+\left(\tilde{\rho} \tilde{\theta}_{m} \nabla_{x} \nabla_{x} \cdot u, \nabla_{x} \theta_{m}\right) \\
& +\left(\tilde{\theta}_{m} \nabla_{x} \nabla_{x} \cdot u, \nabla_{x} \theta_{m}\right)-\epsilon\left(\kappa \nabla_{x} \Delta_{x} \theta_{m}, \nabla_{x} \theta_{m}\right)-\frac{\mu \epsilon}{2}\left(\left(\left(\nabla_{x} u\right)+\left(\nabla_{x} u\right)^{\mathrm{T}}\right)^{2}, \nabla_{x} \theta_{m}\right) \\
& -\lambda \epsilon\left(\left(\nabla_{x} \cdot u\right)^{2}, \nabla_{x} \theta_{m}\right)=0,
\end{aligned}
$$

which further implies

$$
\begin{aligned}
& \left\|\nabla_{x} \rho\right\|_{2}^{2}+\left\|\nabla_{x} \cdot u\right\|_{2}^{2}+\left\|\nabla_{x} \theta_{m}\right\|_{2}^{2}+(\lambda+\mu) \epsilon \int_{0}^{t}\left\|\nabla_{x} \nabla_{x} \cdot u\right\|_{2}^{2} \mathrm{~d} s+\kappa \epsilon \int_{0}^{t}\left\|\nabla_{x}^{2} \theta_{m}\right\|_{2}^{2} \mathrm{~d} s \\
& \lesssim N(0)+\kappa_{0}^{2} \epsilon N(t)+\epsilon \int_{0}^{t}\left\|\nabla_{x}^{2} u\right\|_{2}^{2} \mathrm{~d} s,
\end{aligned}
$$

where we also used the fact $\left\|\nabla_{x}^{2} \theta_{m}\right\|_{2}^{2} \leq C\left\|\Delta_{x} \theta_{m}\right\|_{2}^{2}$. Similarly, it also holds

$$
\begin{aligned}
& \qquad \nabla_{x} \partial_{t}^{\alpha_{0}} \rho\left\|_{2}^{2}+\right\| \nabla_{x} \cdot \partial_{t}^{\alpha_{0}} u\left\|_{2}^{2}+\right\| \nabla_{x} \partial_{t}^{\alpha_{0}} \theta_{m}\left\|_{2}^{2}+(\lambda+\mu) \epsilon \int_{0}^{t}\right\| \nabla_{x} \nabla_{x} \cdot \partial_{t}^{\alpha_{0}} u \|_{2}^{2} \mathrm{~d} s \\
& +\kappa \epsilon \int_{0}^{t}\left\|\nabla_{x}^{2} \partial_{t}^{\alpha_{0}} \theta_{m}\right\|_{2}^{2} \mathrm{~d} s \\
& \lesssim N(0)+\kappa_{0}^{2} \epsilon N(t)+\epsilon \int_{0}^{t}\left\|\nabla_{x}^{2} \partial_{t}^{\alpha_{0}} u\right\|_{2}^{2} \mathrm{~d} s, \\
& \text { for } \alpha_{0} \leq\left[\frac{m_{0}-1}{2}\right] .
\end{aligned}
$$

Step 3. The estimates for $\nabla_{x}^{2} \rho$. For any function $f \in H_{0}^{1}(\Omega)$, we as in the previous step introduce a projection $\mathbb{P}$ and decompose $f=\mathbb{P} f+(I-\mathbb{P}) f$ with $\nabla_{x} \times \mathbb{P} f=0$ and $I f=f$. We now act $\mathbf{P}$ to $(12)_{2}$ to obtain

$$
\left.\mathbf{P}\left\{\rho\left(\partial_{t} u+u \cdot \nabla_{x} u\right)\right\}+\nabla_{x}\left(\rho \theta_{m}\right)=\epsilon\{(\lambda+2 \mu))_{x} \nabla_{x} \cdot u\right\} .
$$

Then $(\lambda+2 \mu) \epsilon^{2}\left(\nabla_{x}^{2}(12)_{1}, \nabla_{x}^{2} \rho\right)+\left(\nabla_{x}(20), \epsilon \nabla_{x}^{2} \rho\right)$ gives rise to

$$
\begin{aligned}
& \frac{(\lambda+2 \mu) \epsilon^{2}}{2} \frac{\mathrm{d}}{\mathrm{d} t}\left\|\nabla_{x}^{2} \rho\right\|_{2}^{2}+(\lambda+2 \mu) \epsilon^{2}\left(\nabla_{x}^{2}\left(\nabla_{x} \rho \cdot u\right), \nabla_{x}^{2} \rho\right) \\
& +(\lambda+2 \mu) \epsilon^{2}\left(\nabla_{x}^{2}\left(\tilde{\rho} \nabla_{x} \cdot u\right), \nabla_{x}^{2} \rho\right)+\epsilon\left(\nabla_{x} \mathbf{P}\left\{\rho\left(\partial_{t} u+u \cdot \nabla_{x} u\right)\right\}, \nabla_{x}^{2} \rho\right) \\
& +\epsilon\left(\theta_{m} \nabla_{x}^{2} \rho, \nabla_{x}^{2} \rho\right)+\epsilon\left(\nabla_{x}^{2} \theta_{m} \rho, \nabla_{x}^{2} \rho\right)+2 \epsilon\left(\nabla_{x} \theta_{m} \nabla_{x} \rho, \nabla_{x}^{2} \rho\right) .
\end{aligned}
$$


Consequently, one has

$$
\epsilon^{2}\left\|\nabla_{x}^{2} \rho\right\|_{2}^{2}+\lambda \epsilon \int_{0}^{t}\left\|\nabla_{x}^{2} \rho\right\|_{2}^{2} \mathrm{~d} s \lesssim N(0)+\kappa_{0}^{2} \epsilon^{2} N(t)+\epsilon \int_{0}^{t}\left\|\left[\partial_{t} \nabla_{x} u, \nabla_{x}^{2} \theta_{m}\right]\right\|_{2}^{2} \mathrm{~d} s,
$$

and a similar calculation leads us to

$$
\begin{aligned}
& \epsilon^{2}\left\|\nabla_{x}^{2} \partial_{t}^{\alpha_{0}} \rho\right\|_{2}^{2}+\lambda \epsilon \int_{0}^{t}\left\|\nabla_{x}^{2} \partial_{t}^{\alpha_{0}} \rho\right\|_{2}^{2} \mathrm{~d} s \\
& \lesssim N(0)+\kappa_{0}^{2} \epsilon^{2} N(t)+\epsilon \int_{0}^{t}\left\|\left[\partial_{t}^{\alpha_{0}+1} \nabla_{x} u, \nabla_{x}^{2} \partial_{t}^{\alpha_{0}} \theta_{m}\right]\right\|_{2}^{2} \mathrm{~d} s, \text { for } \alpha_{0} \leq\left[\frac{m_{0}-2}{2}\right] .
\end{aligned}
$$

Let $m \rightarrow \infty$, we thereupon conclude from (11), (14), (15), (16), (17), (18), (19) and (21) that

$$
\begin{aligned}
& \left\|\partial_{t}^{\alpha_{0}}[\tilde{\rho}, u, \tilde{\theta}](t)\right\|_{2}^{2}+\sum_{\alpha_{0} \leq\left[\left(m_{0}-1\right) / 2\right]}\left\|\nabla_{x} \partial_{t}^{\alpha_{0}}[\tilde{\rho}, u, \tilde{\theta}](t)\right\|_{2}^{2} \\
& +\epsilon^{2} \sum_{\alpha_{0} \leq\left[\left(m_{0}-2\right) / 2\right]}\left\|\nabla_{x}^{2} \partial_{t}^{\alpha_{0}}[\tilde{\rho}, u, \tilde{\theta}](t)\right\|_{2}^{2}+\epsilon^{4} \sum_{\alpha_{0} \leq\left[\left(m_{0}-3\right) / 2\right]}\left\|\nabla_{x}^{3} \partial_{t}^{\alpha_{0}}[u, \tilde{\theta}](t)\right\|_{2}^{2} \\
& +\lambda \sum_{\alpha_{0} \leq\left[m_{0} / 2\right]} \epsilon \int_{0}^{t}\left\|\nabla_{x} \partial_{t}^{\alpha_{0}}[\tilde{\rho}, u, \tilde{\theta}](s)\right\|_{2}^{2} \mathrm{~d} s+\lambda \sum_{\alpha_{0} \leq\left[\left(m_{0}-1\right) / 2\right]} \varepsilon \int_{0}^{t}\left\|\nabla_{x}^{2} \partial_{t}^{\alpha_{0}}[u, \tilde{\theta}](s)\right\|_{2}^{2} \mathrm{~d} s \\
& +\lambda \sum_{\alpha_{0} \leq\left[\left(m_{0}-2\right) / 2\right]} \epsilon \int_{0}^{t}\left\|\nabla_{x}^{2} \partial_{t}^{\alpha_{0}} \tilde{\rho}\right\|_{2}^{2} \mathrm{~d} s \\
& \leq C N(0)+C \epsilon^{2} N(t) .
\end{aligned}
$$

Step 4. Conormal energy estimates. Acting $Z^{\alpha}\left(|\alpha| \leq m_{0}\right)$ to (1), we get

$$
\left\{\begin{array}{l}
Z^{\alpha} \partial_{t} \rho+Z^{\alpha} \nabla_{x} \cdot(\rho u)=0, \\
Z^{\alpha}\left(\rho\left(\partial_{t} u+u \cdot \nabla_{x} u\right)\right)+Z^{\alpha} \nabla_{x} P=\epsilon Z^{\alpha}\{\mu \Delta u+(\lambda+\mu) \nabla \nabla \cdot u\}, P=\rho \theta, \\
\frac{3}{2} Z^{\alpha}\left(\rho\left(\partial_{t} \theta+u \cdot \nabla_{x} \theta\right)\right)+Z^{\alpha}\left(P \nabla_{x} \cdot u\right)=\epsilon Z^{\alpha}\left\{\kappa \Delta \theta+\frac{\mu}{2}\left((\nabla u)+(\nabla u)^{\mathrm{T}}\right)^{2}+\lambda(\nabla \cdot u)^{2}\right\} .
\end{array}\right.
$$

As before, the concormal energy estimates are also divided as the following three steps.

Step 4.1. Basic energy estimates of $Z^{\alpha}[\tilde{\rho}, u, \tilde{\theta}]$. Taking the inner product of $(22)_{1},(22)_{2}$ and $(22)_{3}$ with $Z^{\alpha} \tilde{\rho}, Z^{\alpha} u$ and $\frac{Z^{\alpha} \tilde{\theta}}{\theta}$, respectively, we have

$$
\begin{gathered}
\left((22)_{1}, Z^{\alpha} \tilde{\rho}\right):\left(Z^{\alpha} \partial_{t} \tilde{\rho}, Z^{\alpha} \tilde{\rho}\right)+\left(Z^{\alpha} \nabla_{x} \cdot(\tilde{\rho} u), Z^{\alpha} \tilde{\rho}\right)+\left(Z^{\alpha} \nabla_{x} \cdot u, Z^{\alpha} \tilde{\rho}\right) \\
=\frac{1}{2} \frac{\mathrm{d}}{\mathrm{d} t}\left\|Z^{\alpha} \tilde{\rho}\right\|_{2}^{2}+\left(Z^{\alpha}\left(\nabla_{x} \tilde{\rho} \cdot u\right), Z^{\alpha} \tilde{\rho}\right)+\left(Z^{\alpha}\left(\tilde{\rho} \nabla_{x} \cdot u\right), Z^{\alpha} \tilde{\rho}\right)+\left(Z^{\alpha} \nabla_{x} \cdot u, Z^{\alpha} \tilde{\rho}\right)=0 \\
\left((22)_{2}, Z^{\alpha} u\right):\left(Z^{\alpha}\left(\rho\left(\partial_{t} u+u \cdot \nabla_{x} u\right)\right)+Z^{\alpha} \nabla_{x} P, Z^{\alpha} u\right) \\
=\left(\epsilon Z^{\alpha}\{\mu \Delta u+(\lambda+\mu) \nabla \nabla \cdot u\}, Z^{\alpha} u\right), \\
\left((22)_{3}, \frac{Z^{\alpha} \tilde{\theta}}{\theta}\right):\left(\frac{3}{2} Z^{\alpha}\left(\rho\left(\partial_{t} \theta+u \cdot \nabla_{x} \theta\right)\right)+Z^{\alpha}\left(P \nabla_{x} \cdot u\right), \frac{Z^{\alpha} \tilde{\theta}}{\theta}\right) \\
=\left(\epsilon Z^{\alpha}\left\{\kappa \Delta \theta+\frac{\mu}{2}\left(\left(\nabla_{x} u\right)+\left(\nabla_{x} u\right)^{\mathrm{T}}\right)^{2}+\lambda\left(\nabla_{x} \cdot u\right)^{2}\right\}, \frac{Z^{\alpha} \tilde{\theta}}{\theta}\right)
\end{gathered}
$$

and moreover 


$$
\begin{aligned}
& \left(Z^{\alpha}\left(\rho \partial_{t} u\right), Z^{\alpha} u\right) \\
& =\frac{1}{2} \frac{\mathrm{d}}{\mathrm{d} t} \int_{\Omega} \rho\left(Z^{\alpha} u\right)^{2} d x-\frac{1}{2}\left(\partial_{t} \rho,\left(Z^{\alpha} u\right)^{2}\right)+\sum_{0<\alpha^{\prime} \leq \alpha}\left(Z^{\alpha^{\prime}} \rho Z^{\alpha-\alpha^{\prime}} \partial_{t} u, Z^{\alpha} u\right), \\
& \left(Z^{\alpha}\left(\rho u \cdot \nabla_{x} u\right), Z^{\alpha} u\right) \\
& =-\frac{1}{2} \int_{\Omega} \nabla \cdot(\rho u)\left(Z^{\alpha} u\right)^{2} \mathrm{~d} x+\sum_{0<\alpha^{\prime} \leq \alpha}\left(Z^{\alpha^{\prime}}(\rho u) \cdot Z^{\alpha-\alpha^{\prime}} \nabla u, Z^{\alpha} u\right), \\
& \frac{3}{2}\left(Z^{\alpha}\left(\rho \partial_{t} \theta\right), \frac{Z^{\alpha} \tilde{\theta}}{\theta}\right) \\
& =\frac{3}{4} \frac{\mathrm{d}}{\mathrm{d} t} \int_{\Omega} \frac{\rho}{\theta}\left(Z^{\alpha} \tilde{\theta}\right)^{2} \mathrm{~d} x+\frac{3}{4} \int_{\Omega} \frac{\rho}{\theta^{2}} \partial_{t} \theta\left(Z^{\alpha} \tilde{\theta}\right)^{2} \mathrm{~d} x-\frac{3}{4}\left(\partial_{t} \rho, \frac{1}{\theta}\left(Z^{\alpha} \tilde{\theta}\right)^{2}\right), \\
& \frac{3}{2}\left(Z^{\alpha}\left(\rho u \cdot \nabla_{x} \theta\right), \frac{Z^{\alpha} \tilde{\theta}}{\theta}\right) \\
& =-\frac{3}{4} \int_{\Omega} \nabla_{x} \cdot(\rho u) \frac{1}{\theta}\left(Z^{\alpha} \tilde{\theta}\right)^{2} \mathrm{~d} x+\frac{3}{4} \int_{\Omega} \frac{\rho u}{\theta^{2}} \nabla_{x} \theta\left(Z^{\alpha} \tilde{\theta}\right)^{2} \mathrm{~d} x \\
& \quad+\frac{3}{2} \sum_{0<\alpha^{\prime} \leq \alpha}\left(Z^{\alpha^{\prime}}(\rho u) \cdot Z^{\alpha-\alpha^{\prime}} \nabla_{x} \theta, \frac{1}{\theta} Z^{\alpha} \tilde{\theta}\right) .
\end{aligned}
$$

Consequently, we arrive at

$$
\begin{aligned}
\frac{1}{2} \frac{\mathrm{d}}{\mathrm{d} t}\left\|Z^{\alpha} \tilde{\rho}\right\|_{2}^{2} & +\left(Z^{\alpha}\left(\nabla_{x} \tilde{\rho} \cdot u\right), Z^{\alpha} \tilde{\rho}\right)+\left(Z^{\alpha}\left(\tilde{\rho} \nabla_{x} \cdot u\right), Z^{\alpha} \tilde{\rho}\right)+\left(Z^{\alpha} \nabla_{x} \cdot u, Z^{\alpha} \tilde{\rho}\right)=0, \\
& \frac{1}{2} \frac{\mathrm{d}}{\mathrm{d} t} \int_{\Omega} \rho\left(Z^{\alpha} u\right)^{2} \mathrm{~d} x+\sum_{0<\alpha^{\prime} \leq \alpha}\left(Z^{\alpha^{\prime}} \rho Z^{\alpha-\alpha^{\prime}} \partial_{t} u, Z^{\alpha} u\right) \\
& +\sum_{0<\alpha^{\prime} \leq \alpha}\left(Z^{\alpha^{\prime}}(\rho u) \cdot Z^{\alpha-\alpha^{\prime}} \nabla_{x} u, Z^{\alpha} u\right)+\left(Z^{\alpha}\left(\tilde{\theta} \nabla_{x} \tilde{\rho}\right), Z^{\alpha} u\right) \\
& +\left(Z^{\alpha}\left(\tilde{\rho} \nabla_{x} \tilde{\theta}\right), Z^{\alpha} u\right)+\left(Z^{\alpha} \nabla_{x} \tilde{\rho}, Z^{\alpha} u\right)+\left(Z^{\alpha} \nabla_{x} \tilde{\theta}, Z^{\alpha} u\right) \\
& =\mu \epsilon\left(Z^{\alpha} \Delta u, Z^{\alpha} u\right)+(\lambda+\mu) \epsilon\left(Z^{\alpha} \nabla_{x} \nabla_{x} \cdot u, Z^{\alpha} u\right),
\end{aligned}
$$

and

$$
\begin{aligned}
& \frac{3}{4} \frac{\mathrm{d}}{\mathrm{d} t} \int_{\Omega} \frac{\rho}{\theta}\left(Z^{\alpha} \tilde{\theta}\right)^{2} \mathrm{~d} x+\frac{3}{4} \int_{\Omega} \frac{\rho}{\theta^{2}} \partial_{t} \theta\left(Z^{\alpha} \tilde{\theta}\right)^{2} \mathrm{~d} x+\frac{3}{2} \sum_{0<\alpha^{\prime} \leq \alpha}\left(Z^{\alpha^{\prime}} \rho Z^{\alpha-\alpha^{\prime}} \partial_{t} \theta, \frac{1}{\theta} Z^{\alpha} \tilde{\theta}\right) \\
& +\frac{3}{4} \int_{\Omega} \frac{\rho u}{\theta^{2}} \nabla_{x} \theta\left(Z^{\alpha} \tilde{\theta}\right)^{2} \mathrm{~d} x+\frac{3}{2} \sum_{0<\alpha^{\prime} \leq \alpha}\left(Z^{\alpha^{\prime}}(\rho u) \cdot Z^{\alpha-\alpha^{\prime}} \nabla_{x} \theta, \frac{1}{\theta} Z^{\alpha} \tilde{\theta}\right) \\
& +\left(\tilde{\rho} Z^{\alpha} \nabla_{x} \cdot u, Z^{\alpha} \tilde{\theta}\right)+\left(Z^{\alpha} \nabla_{x} \cdot u, Z^{\alpha} \tilde{\theta}\right)+\sum_{0<\alpha^{\prime} \leq \alpha}\left(Z^{\alpha^{\prime}}(\tilde{\rho} \theta) Z^{\alpha-\alpha^{\prime}} \nabla_{x} \cdot u, \frac{Z^{\alpha} \tilde{\theta}}{\theta}\right) \\
& +\sum_{0<\alpha^{\prime} \leq \alpha}\left(Z^{\alpha^{\prime}} \theta Z^{\alpha-\alpha^{\prime}} \nabla_{x} \cdot u, \frac{Z^{\alpha} \tilde{\theta}}{\theta}\right)=\kappa \epsilon\left(Z^{\alpha} \Delta \theta, \frac{Z^{\alpha} \tilde{\theta}}{\theta}\right) \\
& +\frac{1}{2} \mu \epsilon\left(Z^{\alpha}\left(\left(\nabla_{x} u\right)+\left(\nabla_{x} u\right)^{\mathrm{T}}\right)^{2}, \frac{Z^{\alpha} \tilde{\theta}}{\theta}\right)+\lambda \epsilon\left(Z^{\alpha}\left(\nabla_{x} \cdot u\right)^{2}, \frac{Z^{\alpha} \tilde{\theta}}{\theta}\right) .
\end{aligned}
$$

Taking the summation of (23), (24) and (25), applying Lemmas 1 and 2 and the a priori assumption (5), we then have for some $\lambda>0$ 


$$
\begin{aligned}
& \left\|Z^{\alpha}[\tilde{\rho}, u, \tilde{\theta}](t)\right\|_{2}^{2}+\lambda \epsilon \int_{0}^{t}\left\|Z^{\alpha} \nabla_{x}[u, \tilde{\theta}](s)\right\|_{2}^{2} \mathrm{~d} s \\
& \leq C\left\|Z^{\alpha}[\tilde{\rho}, u, \tilde{\theta}](0, x)\right\|_{2}^{2}+\kappa_{0} \epsilon \int_{0}^{t}\left\|\nabla_{x}[u, \tilde{\theta}](s)\right\|_{H_{c o}^{m_{0}}}^{2} \mathrm{~d} s+\kappa_{0} \epsilon \int_{0}^{t}\left\|\nabla_{x} \tilde{\rho}\right\|_{H_{c o}^{m_{0}-1}}^{2} \mathrm{~d} s,
\end{aligned}
$$

where we also used the following facts

$$
\left(Z^{\alpha} \nabla_{x} \cdot u, Z^{\alpha} \tilde{\rho}\right)+\left(Z^{\alpha} \nabla_{x} \tilde{\rho}, Z^{\alpha} u\right)=0,\left(Z^{\alpha} \nabla_{x} \tilde{\theta}, Z^{\alpha} u\right)+\left(Z^{\alpha} \nabla_{x} \cdot u, Z^{\alpha} \tilde{\theta}\right)=0,
$$

and

$$
\begin{aligned}
& \int_{0}^{t}\left|\left(Z^{\alpha}\left(\nabla_{x} \tilde{\rho} \cdot u\right), Z^{\alpha} \tilde{\rho}\right)\right| \mathrm{d} s \\
& \lesssim \sum_{0 \leq\left|\alpha^{\prime}\right| \leq \frac{|\alpha|}{2}} \kappa_{0} \epsilon^{\frac{1}{2}} \int_{0}^{t}\left(\left\|Z^{\alpha-\alpha^{\prime}} u\right\|_{2}^{2}+\left\|Z^{\alpha} \tilde{\rho}\right\|_{2}^{2}\right) \mathrm{d} s \\
& \quad+\sum_{\frac{|\alpha|}{2}<\left|\alpha^{\prime}\right|<|\alpha|} \kappa_{0} \epsilon \int_{0}^{t}\left(\left\|Z^{\alpha^{\prime}} \nabla_{x} \tilde{\rho}\right\|_{2}^{2}+\left\|Z^{\alpha} \tilde{\rho}\right\|_{2}^{2}\right) \mathrm{d} s+\kappa_{0} \epsilon^{\frac{1}{2}} \int_{0}^{t}\left\|Z^{\alpha} \tilde{\rho}\right\|_{2}^{2} \mathrm{~d} s .
\end{aligned}
$$

Similarly, performing the analogous estimates, one has

$$
\begin{gathered}
\int_{0}^{t}\left|\left(Z^{\alpha} \nabla_{x} \tilde{\rho} \cdot u, Z^{\alpha} \tilde{\rho}\right)\right| \mathrm{d} s \lesssim \sup _{0 \leq s \leq t}\left\|\nabla_{x} \cdot u\right\|_{\infty} \int_{0}^{t} \mid\left\|Z^{\alpha} \tilde{\rho}\right\|_{2}^{2} \mathrm{~d} s \\
\int_{0}^{t}\left|\left(Z^{\alpha}\left(\tilde{\rho} \nabla_{x} \cdot u\right), Z^{\alpha} \tilde{\rho}\right)\right| \mathrm{d} s \leq \sum_{0 \leq \alpha^{\prime} \leq \alpha} \int_{0}^{t}\left|\left(Z^{\alpha^{\prime}} \tilde{\rho} Z^{\alpha-\alpha^{\prime}} \nabla_{x} \cdot u, Z^{\alpha} \tilde{\rho}\right)\right| \mathrm{d} s \\
\lesssim \sum_{0 \leq\left|\alpha^{\prime}\right| \leq \frac{|\alpha|}{2}} \kappa_{0} \epsilon \int_{0}^{t}\left(\left\|Z^{\alpha-\alpha^{\prime}} \nabla_{x} \cdot u\right\|_{2}^{2}+\left\|Z^{\alpha} \tilde{\rho}\right\|_{2}^{2}\right) \mathrm{d} s \\
+\sum_{\frac{|\alpha|}{2}<\left|\alpha^{\prime}\right|<|\alpha|} \kappa_{0} \epsilon^{\frac{1}{2}} \int_{0}^{t}\left(\left\|Z^{\alpha^{\prime}} \tilde{\rho}\right\|_{2}^{2}+\left\|Z^{\alpha} \tilde{\rho}\right\|_{2}^{2}\right) \mathrm{d} s \\
\int_{0<\alpha^{\prime} \leq \alpha}^{t}\left(Z^{\alpha^{\prime}} \rho Z^{\alpha-\alpha^{\prime}} \partial_{t} u, Z^{\alpha} u\right) \mathrm{d} s \lesssim \sum_{0<\alpha^{\prime} \leq \alpha} \kappa_{0} \epsilon \int_{0}^{t}\left(\left\|Z^{\alpha^{\prime}} \rho\right\|_{2}^{2}+\left\|Z^{\alpha} \nabla_{x} u\right\|_{2}^{2}\right) \mathrm{d} s, \\
\int_{0}^{t} \sum_{0<\alpha^{\prime} \leq \alpha}\left|\left(Z^{\alpha^{\prime}}(\rho u) \cdot Z^{\alpha-\alpha^{\prime}} \nabla_{x} u, Z^{\alpha} u\right)\right| \mathrm{d} s \\
\lesssim \sum_{0 \leq\left|\alpha^{\prime \prime}\right| \leq \frac{\left|\alpha^{\prime}\right|}{2} \leq \frac{|\alpha|}{4}} \kappa_{0}^{2} \epsilon^{2} \int_{0}^{t}\left(\left\|Z^{\alpha-\alpha^{\prime}} \nabla_{x} u\right\|_{2}^{2}+\left\|Z^{\alpha} \nabla_{x} u\right\|_{2}^{2}\right) \mathrm{d} s \\
+\sum_{\frac{\left|\alpha^{\prime}\right|}{2}<\left|\alpha^{\prime}\right| \leq\left|\alpha^{\prime}\right| \leq|\alpha|} \kappa_{0}^{2} \epsilon \int_{0}^{t}\left(\left\|Z^{\alpha^{\prime \prime}} u\right\|_{2}^{2}+\left\|Z^{\alpha} u\right\|_{2}^{2}\right) \mathrm{d} s,
\end{gathered}
$$

and

$$
\begin{aligned}
& \int_{0}^{t} \mid\left(Z^{\alpha}\left(\tilde{\theta} \nabla_{x} \tilde{\rho}\right), Z^{\alpha} u\right) \mathrm{d} s \\
& \lesssim \kappa_{0} \epsilon \int_{0}^{t}\left(\left\|Z^{\alpha} \tilde{\rho}\right\|_{2}^{2}+\left\|Z^{\alpha} \nabla_{x} u\right\|_{2}^{2}+\left\|\nabla_{x} \tilde{\theta}\right\|_{2}^{2}\right) \mathrm{d} s \\
& +\sum_{0<\left|\alpha^{\prime}\right| \leq \frac{\alpha}{2}} \kappa_{0} \epsilon \int_{0}^{t}\left(\left\|Z^{\alpha-\alpha^{\prime}} \nabla_{x} \tilde{\rho}\right\|_{2}^{2}+\left\|Z^{\alpha} u\right\|_{2}^{2}\right) \mathrm{d} s \\
& \quad+\sum_{\frac{|\alpha|}{2}\left\langle\left|\alpha^{\prime}\right| \leq|\alpha|\right.} \kappa_{0} \epsilon^{\frac{1}{2}} \int_{0}^{t}\left(\left\|Z^{\alpha^{\prime}} \theta\right\|_{2}^{2}+\left\|\nabla_{x} \tilde{\theta}\right\|_{2}^{2}\right) \mathrm{d} s .
\end{aligned}
$$

To obtain the dissipation of $Z^{\alpha} \nabla_{x} \tilde{\rho}$, we get from the innner product of 
$\epsilon\left((22)_{1}, Z^{\alpha} \nabla_{x} \cdot u\right)$ and $\left((22)_{2}, \frac{Z^{\alpha} \nabla_{x} \tilde{\rho}}{\rho}\right)$ that for any $\eta>0$ and $|\alpha| \leq m_{0}-1$

$$
\begin{aligned}
& -\epsilon\left(Z^{\alpha} u, Z^{\alpha} \nabla_{x} \tilde{\rho}\right)(t)+\lambda \epsilon \int_{0}^{t}\left\|Z^{\alpha} \nabla_{x} \tilde{\rho}\right\|_{2}^{2} \mathrm{~d} s \\
& \leq C \epsilon\left|\left(Z^{\alpha} u, Z^{\alpha} \nabla_{x} \tilde{\rho}\right)(0)\right|+C_{\eta} \epsilon^{3} \int_{0}^{t}\left\|Z^{\alpha} \nabla_{x}^{2} u\right\|_{2}^{2} \mathrm{~d} s \\
& \quad+C\left(\epsilon+\epsilon^{2}\right) \int_{0}^{t}\left\|\nabla_{x}[u, \tilde{\theta}](s)\right\|_{H_{c o}^{m_{0}-1}}^{2} \mathrm{~d} s+C \kappa_{0} \epsilon \int_{0}^{t}\left\|\nabla_{x} \tilde{\rho}\right\|_{H_{c o}^{m_{0}-1}}^{2} .
\end{aligned}
$$

Let $\kappa_{0}$ and $\epsilon$ be suitably small, then (26) and(27) give rise to

$$
\begin{aligned}
& \left\|Z^{\alpha}[\tilde{\rho}, u, \tilde{\theta}](t)\right\|_{2}^{2}-\epsilon\left|\left(Z^{\alpha} u, Z^{\alpha} \nabla_{x} \tilde{\rho}\right)\right|+\lambda \epsilon \int_{0}^{t}\left\|Z^{\alpha} \nabla_{x}[\tilde{\rho}, u, \tilde{\theta}](s)\right\|_{2}^{2} \mathrm{~d} s \\
& \leq C\left\|Z^{\alpha}\left[\tilde{\rho}_{0}, u_{0}, \tilde{\theta}_{0}\right]\right\|_{2}^{2}+C \epsilon\left|\left(Z^{\alpha} u_{0}, Z^{\alpha} \nabla_{x} \tilde{\rho}_{0}\right)\right|+C \epsilon^{3} \int_{0}^{t}\left\|Z^{\alpha} \nabla_{x}^{2} u\right\|_{2}^{2} \mathrm{~d} s \\
& \quad+C \kappa_{0} \epsilon \int_{0}^{t}\left\|\nabla_{x}[u, \tilde{\theta}](s)\right\|_{H_{c o}^{m_{0}}}^{2} \mathrm{~d} s+C \kappa_{0} \epsilon \int_{0}^{t}\left\|\nabla_{x} \tilde{\rho}\right\|_{H_{c o}^{m_{0}-1}}^{2} \mathrm{~d} s \\
& \leq C N(0)+C\left(\epsilon^{2}+\kappa_{0}\right) N(t) .
\end{aligned}
$$

Step 4.2. The energy estimates of $Z^{\alpha} \nabla_{x}[\tilde{\rho}, u, \tilde{\theta}]$ for $|\alpha| \leq m_{0}-1$. As in Step 2, we first decompose $Z^{\alpha} u$ as $Z^{\alpha} u=Z^{\alpha} u^{1}+Z^{\alpha} u^{2}$ with $Z^{\alpha} u^{1}=\nabla_{x} \phi, Z^{\alpha} u^{2}=\nabla_{x} \times \psi$ and $\left.Z^{\alpha} u^{2}\right|_{\partial \Omega}=0$. Moreover, we set $Z^{\alpha} \theta_{m}(t, x)-1=\sum_{k=1}^{m} a_{k}(t) p_{k}(x)$ with $p_{k}(x) \in H_{0}^{1}(\Omega)(k=1,2, \cdots)$ being the eigenvalues of the operator $-\Delta_{x}$, i.e.

$$
\left\{\begin{array}{l}
-\Delta_{x} p_{k}=\lambda_{k} p_{k}, x \in \Omega, \\
p_{k}(x)=0, x \in \partial \Omega
\end{array}\right.
$$

where $0<\lambda_{1} \leq \lambda_{2} \leq \cdots$.

We now approximate (22) as

$$
\left\{\begin{array}{l}
Z^{\alpha} \partial_{t} \rho+Z^{\alpha} \nabla_{x} \cdot(\rho u)=0, \\
Z^{\alpha}\left(\rho\left(\partial_{t} u+u \cdot \nabla_{x} u\right)+\nabla_{x}\left(\rho \theta_{m}\right)\right)=\epsilon Z^{\alpha}\left\{\mu \Delta u+(\lambda+\mu) \nabla_{x} \nabla_{x} \cdot u\right\}, \\
\frac{3}{2} Z^{\alpha}\left(\rho\left(\partial_{t} \theta_{m}+u \cdot \nabla_{x} \theta_{m}\right)+\rho \theta_{m} \nabla_{x} \cdot u\right)=\epsilon Z^{\alpha}\left\{\kappa \Delta \theta_{m}+\frac{\mu}{2}\left(\left(\nabla_{x} u\right)+\left(\nabla_{x} u\right)^{\mathrm{T}}\right)^{2}+\lambda\left(\nabla_{x} \cdot u\right)^{2}\right\}, \\
Z^{\alpha}\left[\rho, u, \theta_{m}\right](0, x)=Z^{\alpha}\left[\rho_{0}, u_{0}, \theta_{0}\right](x), \\
\left.Z^{\alpha}\left[u, \theta_{m}\right]\right]_{\partial \Omega}=[0,1] .
\end{array}\right.
$$

Note that here $\left[Z^{\alpha} \rho, Z^{\alpha} u\right] \stackrel{\text { def }}{=}\left[\rho_{m}, u_{m}\right]$ also depend on $m$, we drop the subscript $m$ for brevity.

Noticing that $\nabla_{x} \cdot Z^{\alpha} u^{2}=0$ and $\left(Z^{\alpha} u^{1}, Z^{\alpha} u^{2}\right)=0$, taking the inner product of (29) ${ }_{2}$ with $Z^{\alpha} \partial_{t} u^{2}$ and integrating the resulting equation with respect to $t$, one has

$$
\begin{aligned}
& \epsilon\left(Z^{\alpha} \nabla_{x} \times u^{2}, Z^{\alpha} \nabla_{x} \times u^{2}\right)+\lambda \int_{0}^{t}\left\|Z^{\alpha} \partial_{t} u^{2}\right\|_{2}^{2} \mathrm{~d} s \\
& \leq C \epsilon\left|\left(Z^{\alpha} \nabla_{x} \times u_{0}^{2}, Z^{\alpha} \nabla_{x} \times u_{0}^{2}\right)\right|+C \int_{0}^{t}\left\|\left.Z^{\alpha}\left(\tilde{\rho} \partial_{t} u\right)\right|_{2} ^{2} \mathrm{~d} s+C \int_{0}^{t}\right\| Z^{\alpha}\left(\rho u \cdot \nabla_{x} u\right) \|_{2}^{2} \mathrm{~d} s \\
& \leq C \epsilon\left|\left(Z^{\alpha} \nabla_{x} \times u_{0}^{2}, Z^{\alpha} \nabla_{x} \times u_{0}^{2}\right)\right|+C \kappa_{0}^{2} \epsilon^{2} \int_{0}^{t}\left\|\nabla_{x}[\tilde{\rho}, u]\right\|_{H_{c o}^{m_{0}-1}}^{2} \mathrm{~d} s \\
& \leq C \epsilon N(0)+C \kappa_{0}^{2} \epsilon N(t) .
\end{aligned}
$$


Next, we consider the following elliptic problems:

$\left\{\begin{array}{l}\epsilon Z^{\alpha} \nabla_{x} \cdot u=-\epsilon Z^{\alpha} \partial_{t} \rho-\epsilon Z^{\alpha}\left(\tilde{\rho} \nabla_{x} \cdot u\right)-\epsilon Z^{\alpha}\left(\nabla_{x} \rho \cdot u\right) \stackrel{\text { def }}{=} h_{1}^{\prime}, \\ -\mu \epsilon Z^{\alpha} \Delta u+Z^{\alpha} \nabla_{x}\left[\left(\rho \theta_{m}\right)+\partial_{t} \phi-\epsilon(\lambda+\mu) \nabla_{x} \cdot u\right]=-Z^{\alpha} \partial_{t} u^{2}-Z^{\alpha}\left(\tilde{\rho} \partial_{t} u\right)-Z^{\alpha}\left(\rho u \cdot \nabla_{x} u\right) \stackrel{\text { def }}{=} h_{2}^{\prime}, \\ \left.Z^{\alpha} u\right|_{\partial \Omega}=0 .\end{array}\right.$

Since $\alpha_{0} \leq\left[m_{0}-2\right]$, it follows

$$
\begin{aligned}
\epsilon^{2}\left\|Z^{\alpha} \nabla_{x}^{2} u\right\|_{2}^{2} \lesssim & \left\|\nabla_{x} h_{1}^{\prime}\right\|_{2}^{2}+\left\|h_{2}^{\prime}\right\|_{2}^{2} \\
\lesssim & \epsilon^{2}\left\|Z^{\alpha} \partial_{t} \nabla_{x} \tilde{\rho}\right\|_{2}^{2}+\kappa_{0}^{2} \epsilon^{3}\left\|\nabla_{x}[\tilde{\rho}, u]\right\|_{H_{c o}^{m_{0}-2}}^{2}+\kappa_{0}^{2} \epsilon^{4}\left\|\nabla_{x}^{2}[\tilde{\rho}, u]\right\|_{H_{c o}^{m_{0}-2}}^{2} \\
& +\left\|Z^{\alpha} \partial_{t} u^{2}\right\|_{2}^{2}+\kappa_{0}^{2} \epsilon^{2}\left\|\nabla_{x}[\tilde{\rho}, u]\right\|_{H_{c o}^{m_{0}-2}}^{2} .
\end{aligned}
$$

Combing (28), (30) and (31), we arrive at

$$
\begin{aligned}
& \left(Z^{\alpha} \nabla_{x} \times u^{2}, Z^{\alpha} \nabla_{x} \times u^{2}\right)+\frac{\lambda}{\epsilon} \int_{0}^{t}\left\|Z^{\alpha} \partial_{t} u^{2}\right\|_{2}^{2} \mathrm{~d} s+\lambda \epsilon \int_{0}^{t}\left\|Z^{\alpha} \nabla_{x}^{2} u\right\|_{2}^{2} \mathrm{~d} s \\
& \leq C N(0)+C\left(\kappa_{0}^{2}+\epsilon^{2}\right) N(t) .
\end{aligned}
$$

Moreover, by using

$$
-\mu \epsilon Z^{\alpha} \Delta u-\epsilon(\lambda+\mu) Z^{\alpha} \nabla_{x} \nabla_{x} \cdot u=-Z^{\alpha}\left(\rho \partial_{t} u\right)-Z^{\alpha}\left(\rho u \cdot \nabla_{x} u\right)-Z^{\alpha} \nabla_{x}\left(\rho \theta_{m}\right),
$$

and

$$
\begin{aligned}
-\kappa \epsilon Z^{\alpha} \Delta \theta= & -\frac{3}{2} Z^{\alpha}\left(\rho\left(\partial_{t} \theta_{m}+u \cdot \nabla_{x} \theta_{m}\right)+\rho \theta_{m} \nabla_{x} \cdot u\right) \\
& +\epsilon Z^{\alpha}\left\{\frac{\mu}{2}\left(\left(\nabla_{x} u\right)+\left(\nabla_{x} u\right)^{\mathrm{T}}\right)^{2}+\lambda\left(\nabla_{x} \cdot u\right)^{2}\right\},
\end{aligned}
$$

the standard elliptic estimates implies

$$
\begin{gathered}
\epsilon^{4}\left\|Z^{\alpha} \nabla_{x}^{3} u\right\|_{2}^{2} \lesssim \epsilon^{2}\left\|Z^{\alpha} \partial_{t} u\right\|_{H^{1}}^{2}+\epsilon^{2}\left\|Z^{\alpha} \nabla_{x}\left[\tilde{\rho}, \theta_{m}\right]\right\|_{H^{1}}^{2}+\kappa_{0}^{2} \epsilon^{3}\left\|Z^{\alpha} \nabla_{x} u\right\|_{H^{1}}^{2}, \\
\epsilon^{2}\left\|Z^{\alpha} \nabla_{x}^{2} \theta_{m}\right\|_{2}^{2} \lesssim\left\|\left.\left[Z^{\alpha} \partial_{t} \theta_{m}, Z^{\alpha} \nabla_{x} u\right]\right|_{2} ^{2}+\kappa_{0}^{2} \epsilon^{2}\right\| Z^{\alpha} \nabla_{x}\left[u, \theta_{m}\right] \|_{2}^{2}, \\
\epsilon^{4}\left\|Z^{\alpha} \nabla_{x}^{3} \theta_{m}\right\|_{2}^{2} \lesssim \epsilon^{2}\left\|\left[Z^{\alpha} \partial_{t} \theta_{m}, Z^{\alpha} \nabla_{x} u\right]\right\|_{H^{1}}^{2}+\kappa_{0}^{2} \epsilon^{4}\left\|Z^{\alpha} \nabla_{x}\left[u, \theta_{m}\right]\right\|_{H^{1}}^{2}
\end{gathered}
$$

Next, $\left(\nabla_{x}(29)_{1}, Z^{\alpha} \nabla_{x} \rho\right)-\left((29)_{2}, Z^{\alpha} \nabla_{x} \nabla_{x} \cdot u\right)+\left(\nabla_{x}(29)_{3}, Z^{\alpha} \nabla_{x} \theta_{m}\right)$ yields

$$
\begin{aligned}
& \frac{1}{2} \frac{\mathrm{d}}{\mathrm{d} t}\left\|Z^{\alpha} \nabla_{x} \rho\right\|_{2}^{2}+\frac{1}{2} \frac{\mathrm{d}}{\mathrm{d} t}\left\|\sqrt{\rho} Z^{\alpha} \nabla_{x} \cdot u\right\|_{2}^{2}+\frac{3}{4} \frac{\mathrm{d}}{\mathrm{d} t}\left\|\sqrt{\rho} Z^{\alpha} \nabla_{x} \theta_{m}\right\|_{2}^{2} \\
& +\left(Z^{\alpha} \nabla_{x}\left(\nabla_{x} \rho \cdot u\right), Z^{\alpha} \nabla_{x} \rho\right)+\left(Z^{\alpha} \nabla_{x}\left(\tilde{\rho} \nabla_{x} \cdot u\right), Z^{\alpha} \nabla_{x} \rho\right) \\
& -\frac{1}{2}\left(\rho_{t} Z^{\alpha} \nabla_{x} \cdot u, Z^{\alpha} \nabla_{x} \cdot u\right)+\sum_{0<\alpha^{\prime} \leq \alpha}\left(Z^{\alpha^{\prime}} \rho Z^{\alpha-\alpha^{\prime}} \nabla_{x} \cdot \partial_{t} u, Z^{\alpha} \nabla_{x} u\right) \\
& +\left(Z^{\alpha}\left(\nabla_{x} \rho \partial_{t} u\right), Z^{\alpha} \nabla_{x} \cdot u\right)+\left(Z^{\alpha} \nabla_{x} \cdot\left(\rho u \cdot \nabla_{x} u\right), Z^{\alpha} \nabla_{x} \cdot u\right) \\
& -\left(Z^{\alpha}\left(\tilde{\theta}_{m} \nabla_{x} \rho\right), Z^{\alpha} \nabla_{x} \nabla_{x} \cdot u\right)-\left(Z^{\alpha}\left(\tilde{\rho} \nabla_{x} \theta_{m}\right), Z^{\alpha} \nabla_{x} \nabla_{x} \cdot u\right)
\end{aligned}
$$




$$
\begin{aligned}
& +\epsilon\left(Z^{\alpha}\left\{\mu \Delta u+(\lambda+\mu) \nabla_{x} \nabla_{x} \cdot u\right\}, Z^{\alpha} \nabla_{x} \nabla_{x} \cdot u\right)-\frac{3}{4}\left(\partial_{t} Z^{\alpha} \nabla_{x} \theta_{m}, Z^{\alpha} \nabla_{x} \theta_{m}\right) \\
& +\frac{3}{2}\left(Z^{\alpha}\left(\nabla_{x} \rho \partial_{t} \theta_{m}\right), Z^{\alpha} \nabla_{x} \theta_{m}\right)+\frac{3}{2} \sum_{0<\alpha^{\prime} \leq \alpha}\left(Z^{\alpha^{\prime}} \rho Z^{\alpha-\alpha^{\prime}} \nabla_{x} \partial_{t} \theta_{m}, Z^{\alpha} \nabla_{x} \theta_{m}\right) \\
& +\frac{3}{2}\left(Z^{\alpha} \nabla_{x}\left(\rho u \cdot \nabla_{x} \theta_{m}\right), Z^{\alpha} \nabla_{x} \theta_{m}\right)+\left(Z^{\alpha}\left(\nabla_{x}\left(\rho \theta_{m}\right) \nabla_{x} \cdot u\right), Z^{\alpha} \nabla_{x} \theta_{m}\right) \\
& +\left(Z^{\alpha}\left(\tilde{\rho} \tilde{\theta}_{m} \nabla_{x} \nabla_{x} \cdot u\right), Z^{\alpha} \nabla_{x} \theta_{m}\right)+\left(Z^{\alpha}\left(\tilde{\rho} \nabla_{x} \nabla_{x} \cdot u\right), Z^{\alpha} \nabla_{x} \theta_{m}\right) \\
& +\left(Z^{\alpha}\left(\tilde{\theta}_{m} \nabla_{x} \nabla_{x} \cdot u\right), Z^{\alpha} \nabla_{x} \theta_{m}\right)-\epsilon\left(\kappa Z^{\alpha} \nabla_{x} \Delta \theta_{m}, Z^{\alpha} \nabla_{x} \theta_{m}\right) \\
& -\frac{\mu \epsilon}{2}\left(Z^{\alpha} \nabla_{x}\left(\left(\nabla_{x} u\right)+\left(\nabla_{x} u\right)^{\mathrm{T}}\right)^{2}, Z^{\alpha} \nabla_{x} \theta_{m}\right)-\lambda \epsilon\left(Z^{\alpha}\left(\nabla_{x} \cdot u\right)^{2}, Z^{\alpha} \nabla_{x} \theta_{m}\right)=0,
\end{aligned}
$$

which further implies

$$
\begin{aligned}
& \left\|Z^{\alpha} \nabla_{x} \rho\right\|_{2}^{2}+\left\|Z^{\alpha} \nabla_{x} \cdot u\right\|_{2}^{2}+\left\|Z^{\alpha} \nabla_{x} \theta_{m}\right\|_{2}^{2}+\lambda \epsilon \int_{0}^{t}\left\|Z^{\alpha} \nabla_{x} \nabla_{x} \cdot u\right\|_{2}^{2}+\left\|Z^{\alpha} \nabla_{x}^{2} \theta_{m}\right\|_{2}^{2} \mathrm{~d} s \\
& \lesssim N(0)+\kappa_{0}^{2} \epsilon N(t)+\epsilon \int_{0}^{t}\left\|Z^{\alpha} \nabla_{x}^{2} u\right\|_{2}^{2} \mathrm{~d} s,
\end{aligned}
$$

for $\alpha \leq\left[m_{0}-1\right]$.

Step 4.3. The estimates of $Z^{\alpha} \nabla_{x}^{2} \rho$ with $\alpha \leq\left[m_{0}-2\right]$. For any function $g \in H_{0}^{1}(\Omega)$, we introduce a projection $\mathbb{P}$ and decompose $g=\mathbb{P} g+(I-\mathbb{P}) g$ with $\nabla_{x} \times \mathbb{P g}=0$ and $I g=g$. We now act $\mathbb{P}$ to $(29)_{2}$ to obtain

$$
\mathbb{P}\left\{Z^{\alpha}\left(\rho \partial_{t} u+u \cdot \nabla_{x} u\right)\right\}+Z^{\alpha} \nabla_{x}\left(\rho \theta_{m}\right)=\epsilon\left\{(\lambda+2 \mu) Z^{\alpha} \nabla_{x} \nabla_{x} \cdot u\right\} .
$$

Then $(\lambda+2 \mu) \epsilon^{2}\left(\nabla_{x}^{2}(29)_{1}, Z^{\alpha} \nabla_{x}^{2} \rho\right)+\left(\nabla_{x}(37), \epsilon Z^{\alpha} \nabla_{x}^{2} \rho\right)$ gives rise to

$$
\begin{aligned}
& (\lambda+2 \mu) \frac{\epsilon^{2}}{2} \frac{\mathrm{d}}{\mathrm{d} t}\left\|Z^{\alpha} \nabla_{x}^{2} \rho\right\|_{2}^{2}+\epsilon^{2}(\lambda+2 \mu)\left(Z^{\alpha} \nabla_{x}^{2}\left(\nabla_{x} \rho \cdot u\right), Z^{\alpha} \nabla_{x}^{2} \rho\right) \\
& +(\lambda+2 \mu) \epsilon^{2}\left(Z^{\alpha} \nabla_{x}^{2}\left(\tilde{\rho} \nabla_{x} \cdot u\right), Z^{\alpha} \nabla_{x}^{2} \rho\right)+(\lambda+2 \mu) \epsilon^{2}\left(Z^{\alpha} \nabla_{x}^{2} \nabla_{x} \cdot u, Z^{\alpha} \nabla_{x}^{2} \rho\right) \\
& +\epsilon\left(\nabla_{x} \mathbb{P}\left\{Z^{\alpha}\left(\rho \partial_{t} u+\rho u \cdot \nabla_{x} u\right)\right\}, Z^{\alpha} \nabla_{x}^{2} \rho\right)+\epsilon\left(Z^{\alpha}\left(\theta_{m} \nabla_{x}^{2} \rho\right), Z^{\alpha} \nabla_{x}^{2} \rho\right) \\
& +\epsilon\left(Z^{\alpha}\left(\nabla_{x}^{2} \theta_{m} \rho\right), Z^{\alpha} \nabla_{x}^{2} \rho\right)+2 \epsilon\left(Z^{\alpha}\left(\nabla_{x} \theta_{m} \nabla_{x} \rho\right), Z^{\alpha} \nabla_{x}^{2} \rho\right) \\
& -\epsilon^{2}(\lambda+2 \mu)\left(Z^{\alpha} \nabla_{x} \nabla_{x} \nabla_{x} \cdot u, Z^{\alpha} \nabla_{x}^{2} \rho\right) .
\end{aligned}
$$

Consequently, one has

$$
\begin{aligned}
& \epsilon^{2}\left\|Z^{\alpha} \nabla_{x}^{2} \rho\right\|_{2}^{2}+\lambda \epsilon \int_{0}^{t}\left\|Z^{\alpha} \nabla_{x}^{2} \rho\right\|_{2}^{2} \mathrm{~d} s \\
& \lesssim N(0)+\kappa_{0}^{2} \epsilon^{2} N(t)+\epsilon \int_{0}^{t}\left\|\left[\partial_{t} \nabla_{x} u, \nabla_{x}^{2} \theta_{m}\right]\right\|_{H_{c o}^{m_{0}-2}}^{2} .
\end{aligned}
$$

Let $m \rightarrow \infty$, we thereupon conclude from (28), (31), (32), (33), (34), (35), (36) and (38) that

$$
\begin{aligned}
& \left\|Z^{\alpha}[\tilde{\rho}, u, \tilde{\theta}](t)\right\|_{2}^{2}+\sum_{|\alpha| \leq m_{0}-1}\left\|\nabla_{x} Z^{\alpha}[\tilde{\rho}, u, \tilde{\theta}](t)\right\|_{2}^{2}+\epsilon^{2} \sum_{|\alpha| \leq m_{0}-2}\left\|\nabla_{x}^{2} Z^{\alpha}[\tilde{\rho}, u, \tilde{\theta}](t)\right\|_{2}^{2} \\
& +\epsilon^{4} \sum_{|\alpha| \leq m_{0}-3}\left\|\nabla_{x}^{3} Z^{\alpha}[u, \tilde{\theta}](t)\right\|_{2}^{2}+\lambda \sum_{|\alpha| \leq m_{0}} \epsilon \int_{0}^{t}\left\|\nabla_{x} Z^{\alpha}[\tilde{\rho}, u, \tilde{\theta}](s)\right\|_{2}^{2} d s \\
& +\lambda \sum_{|\alpha| \leq m_{0}-1} \epsilon \int_{0}^{t}\left\|\nabla_{x}^{2} Z^{\alpha}[u, \tilde{\theta}](s)\right\|_{2}^{2} \mathrm{~d} s+\lambda \sum_{|\alpha| \leq m_{0}-2} \epsilon \int_{0}^{t}\left\|\nabla_{x}^{2} Z^{\alpha} \tilde{\rho}(s)\right\|_{2}^{2} d s \\
& \leq C N(0)+C \epsilon^{2} N(t) .
\end{aligned}
$$


Finally, we close our estimates by letting $\epsilon$ be suitably small. This ends the proof of Theorem 1.

\section{Conflicts of Interest}

The authors declare no conflicts of interest regarding the publication of this paper.

\section{References}

[1] Galdi, G.P. (2012) Navier-Stokes Equations: A Mathematical Analysis. In: Meyers, R., Eds., Mathematics of Complexity and Dynamical Systems, Spring, New York, NY.

[2] Bardos, C., Golse, F. and Levermore, D.C. (1991) Fluid Dynamic Limits of the Kinetic Equation. I. Formal Derivation. Journal of Statistical Physics, 63, 323-344. https://doi.org/10.1007/BF01026608

[3] Liu, S.-Q., Yang, T. and Zhao, H.-J. (2014) Compressible Navier-Stokes Approximation to the Boltzmann Equation. Journal of Differential Equations, 256, 3770-3816. https://doi.org/10.1016/j.jde.2014.02.020

[4] Duan, R.-J. and Liu, S.-Q. Compressible Navier-Stokes Approximation for the Boltzmann Equation in Bounded Domains. arXiv:1806.09796

[5] Wang, Y. (2016) Uniform Regularity and Vanishing Dissipation Limit for the Full Compressible Navier-Stokes System in Three Dimensional Bounded Domain. Archive for Rational Mechanics and Analysis, 221, 1345-1415. https://doi.org/10.1007/s00205-016-0989-8

[6] Wang, Y., Xin, Z.-P. and Yong, Y. (2015) Uniform Regularity and Vanishing Viscosity Limit for the Compressible Navier-Stokes with General Navier-Slip Boundary Conditions in Three-Dimensional Domains. SIAM Journal on Mathematical Analysis, 47, 4123-4191. https://doi.org/10.1137/151003520

[7] Masmoudi, N. and Rousset, F. (2017) Uniform Regularity and Vanishing Viscosity Limit for the Free Surface Navier-Stokes Equations. Archive for Rational Mechanics and Analysis, 223, 301-417. https://doi.org/10.1007/s00205-016-1036-5

[8] Matsumura, A. and Nishida, T. (1980) The Initial Value Problem for the Equations of Motion of Viscous and Heat-Conductive Gases. Journal of Mathematics of Kyoto University, 26, 67-104. https://doi.org/10.1215/kjm/1250522322

[9] Matsumura, A. and Nishida, T. (1983) Initial-Boundary Value Problems for the Equations of Motion of Compressible Viscous and Heat-Conductive Fluids. Communications in Mathematical Physics, 89, 445-464. https://doi.org/10.1007/BF01214738

[10] Huang, X.-D., Li, J. and Xin, Z.-P. (2012) Global Well-Posedness of Classical Solutions with Large Oscillations and Vacuum to the Three-Dimensional Isentropic Compressible Navier-Stokes Equations. Communications on Pure and Applied Mathematics, 65, 549-585. https://doi.org/10.1002/cpa.21382

[11] Ukai, S., Yang, T. and Zhao, H.-J. (2006) Convergence Rate for the Compressible Navier-Stokes Equations with External Force. Journal of Hyperbolic Differential Equations, 3, 561-574. https://doi.org/10.1142/S0219891606000902

[12] Duan, R.-J., Liu, H.-X., Ukai, S. and Yang, T. (2007) Optimal $L^{p}-L^{q}$ Convergence Rates for the Compressible Navier-Stokes Equations with Potential Force. 
Journal of Differential Equations, 238, 220-233.

https://doi.org/10.1016/j.jde.2007.03.008

[13] Lions, P.L. (1998) Mathematical Topics in Fluid Mechanics. In: Compressible Models. Oxford Lecture Series in Mathematics and Its Applications, Vol. 2. 10, Clarendon, University Press, New York. 